\title{
Development of Image Analysis Techniques \\ as a Tool to Detect and Quantify \\ Morphological Changes in Anaerobic Sludge: \\ I. Application to a Granulation Process
}

\author{
P. Araya-Kroff, ${ }^{1}$ A.L. Amaral, ${ }^{1}{ }^{*}$ L. Neves, ${ }^{1}$ E.C. Ferreira, ${ }^{1}$ M.-N. Pons, ${ }^{2}$ \\ M. Mota, ${ }^{1}$ M.M. Alves ${ }^{1}$ \\ ${ }^{1}$ Centro de Engenharia Biológica, Universidade do Minho, 4710-057 Braga, \\ Portugal; telephone: + 351253604 402; fax: + 351253678 986; \\ e-mail: madalena.alves@deb.uminho.pt \\ ${ }^{2}$ Laboratoire des Sciences du Génie Chimique, CNRS-ENSIC-INPL, BP 451, \\ F-54001 Nancy Cedex, France
}

Received 22 October 2003; accepted 9 March 2004

Published online 17 June 2004 in Wiley InterScience (www.interscience.wiley.com). DOI: 10.1002/bit.20207

\begin{abstract}
Image analysis techniques were developed and applied to quantify the process of anaerobic granulation in an expanded granular sludge blanket reactor (EGSB) fed with a synthetic substrate based on glucose [60-30\% COD (chemical oxygen demand)] and volatile fatty acids (40-70\% COD) over 376 days. In a first operation period that lasted 177 days, the aggregation of dispersed sludge was quantitatively monitored through the recognition and quantification of aggregates and filaments. A parameter defined as the ratio between the filaments' length and the aggregates projected area $(L f A)$ has proven to be sensitive to detect changes in the aggregation status of the anaerobic sludge. The aggregation time-defined as the moment when a balance between filaments' length and aggregates' size was established-was recognized through the $L f A$. The percentage of projected area of aggregates within three size ranges $(0.01-0.1 \mathrm{~mm}, 0.1-1 \mathrm{~mm}$, and $>1 \mathrm{~mm}$, equivalent diameter) reflected the granular size spectrum during the aggregation process. When sudden increases on the upflow velocity and on the organic loading rate were applied to the previously formed granules, the developed image analysis techniques revealed to be good indicators of granular sludge stability, since they were sensitive to detected filaments release, fragmentation, and erosion that usually leads to washout. The specific methanogenic activities in the presence of acetate, propionate, butyrate, and $\mathrm{H}_{2} / \mathrm{CO}_{2}$ increased along the operation, particularly relevant was the sudden increase in the specific hydrogenophilic activity, immediately after the moment recognized as aggregation time. (c) 2004 Wiley Periodicals, Inc.
\end{abstract}

Correspondence to: M.M. Alves

*Present address: Departamento de Tecnologia Química, Escola Superior de Tecnologia e de Gestão, Instituto Politécnico de Bragança, Campus de Santa Apolónia, Apartado 1038, 5301-854 Bragança, Portugal

Contract grant sponsors: Fundação para a Ciência e a Tecnologia (Portugal); ICCTI (Portugal); Ambassade de France (Portugal)

Contract grant numbers: SFRH/BD/3187/2000; PRAXIS XXI/BD/ 20325/99; POCTI/1999/CTA736524; Project No. 203 B4
Keywords: expanded granular sludge blanket reactor (EGSB); aggregation time; granules; image analysis; methanogenic activity

\section{INTRODUCTION}

Since their development in the early 1980s, anaerobic sludge bed reactors based on granular biomass, have represented a significant technological advance for industrial anaerobic wastewater treatment (van Lier et al., 2001). The upflow anaerobic sludge blanket (UASB) reactor, which has been successfully applied for the treatment of several types of effluents (Frankin, 2001), operates typically at superficial velocities (SV) of $2 \mathrm{~m} / \mathrm{h}$ and can accommodate organic loading rates (OLR) up to $10 \mathrm{kgCOD} \mathrm{m}^{3} \mathrm{~d}$ $(\mathrm{COD}=$ chemical oxygen demand). This reactor configuration evolved to the EGSB (expanded granular sludge blanket) reactor, which operates at $\mathrm{SV}$ up to $10 \mathrm{~m} / \mathrm{h}$ and OLR up to $40 \mathrm{kgCOD} \mathrm{m}^{3} \mathrm{~d}$. Both reactor configurations are based on the differentiation between the hydraulic retention time and the solids retention time, which is achieved by the use of dense granular sludge.

Several hypotheses have been proposed for the granulation process, some of them focusing on substrate composition and operating conditions, while others address the thermodynamic aspects. In general, it is widely accepted that the microorganisms involved play an important role. Wiegant and de Man (1986) pointed out that Methanosaeta is of vital importance for the formation of the initial web structures around which the aggregation process takes place. In their theory, the granulation process is divided into two stages: a first stage, in which enrichment on the acetoclastic filamentous Methanosaeta has to be ensured to form granulation nuclei, and a second stage, when the granule grows over the previously formed nuclei, ElMamouni et al. (1995) stated that Methanosaeta and the 
syntrophic species were the relevant populations for the granulation process and Morvai et al. (1992) reported that the presence of Methanosaeta guarantees a rapid granulation. The presence of Methanosaeta in the granulation nuclei is also referred by Macleod et al. (1990) who proposed a multilayered structure of granules formed by successive attachments of bacteria as the process stimulated by the syntrophic relationship.

Besides the microbial factor, the "selection pressure" factor reported by Hulshoff Pol (1989) promotes the washout of nonattached cells and small fragments, and selects for the more dense aggregates that will remain inside the reactor and will grow by means of successive cell attachment, growth, and matrix mineralization. It is one of the most important factors responsible for the granulation process.

To understand the granulation process, it is important to know how to promote it, and how to recognize specific moments in the process, for instance the moment when granules' maturity is achieved (Singh et al., 1998). Therefore, it is essential to have sensitive indicators to identify these moments and to quantify and control the size spectrum of granular sludge in anaerobic reactors (Lettinga and Hulshoff Pol, 2002).

Classical methods to characterize microbial aggregates and filaments in wastewater treatment processes were based on manual counting techniques and have been primarily developed for activated sludge processes to alert for bulking problems (Jenkins et al., 1993). Manual counting techniques are, however, rather laborious, imprecise, and time-consuming methods, which make them difficult to implement in a quantitative way.

The technological advances and the decrease of computation costs gave the opportunity of techniques such as quantitative image analysis to be used for process monitoring. These techniques have already been applied in the quantitative morphological characterization of anaerobic sludge (Alves et al., 2000a; Alves et al., 2000b). The main difficulty is to define and accurately measure the most suit- able parameters that are sensitive to the granulation, granules maturation, or granules deterioration processes. Such parameters should be good indicators of sludge bed stability, a crucial factor for UASB/EGSB long-run operation.

Our aim in the present study was to develop and apply quantitative image analysis techniques to identify critical events of the anaerobic granulation process, to propose morphological parameters sensitive to the aggregation status of anaerobic sludge, and to discuss the usefulness of quantitative image analysis to monitor the structural and morphological stability of anaerobic granular sludge. More specifically, the relative abundance of filaments, either free or protruding from dense biomass (aggregates), with respect to the latter, will be quantified (Fig. 1).

\section{MATERIALS AND METHODS}

\section{EGSB Reactor and Operation Mode}

The expanded granular sludge bed (EGSB) reactor was made of plexiglas with a volume of $11.5 \mathrm{~L}$, a height of $2.22 \mathrm{~m}$, and a height-to-diameter ratio of 27 . The reactor set-up included an external settler where the washed out biomass was collected, two recirculation ports, and three sampling ports alongside its height (Fig. 2). The temperature was kept at $37 \pm 1{ }^{\circ} \mathrm{C}$ by means of an external jacket for water circulation.

The reactor was operated during 376 days. The operation of the EGSB reactor was divided into two operating periods (OP I, between days 0 and 177 and OP II, between days 211 and 376). Between days 178 and 210, the operation was stopped and biomass was kept at $4{ }^{\circ} \mathrm{C}$. In the first operating period, the superficial upflow velocity was kept below $4 \mathrm{~m} / \mathrm{h}$ to prevent the washout of growing granulation nuclei, but still high enough to maintain a mixing pattern that promoted the contact between the aggregates. During the second operation period organic loading rates (OLR) up to

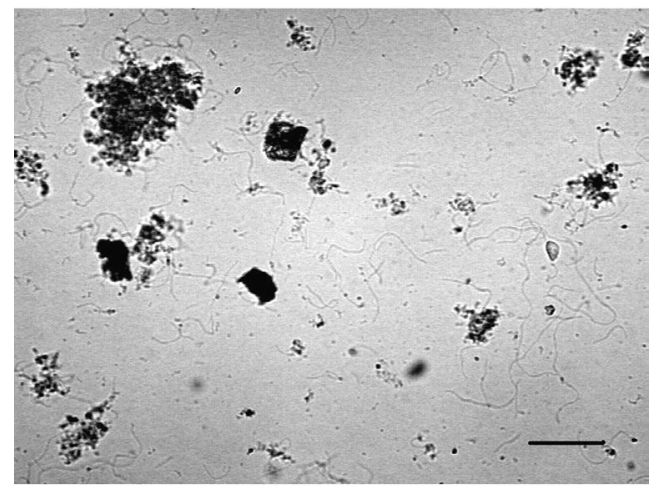

(a)

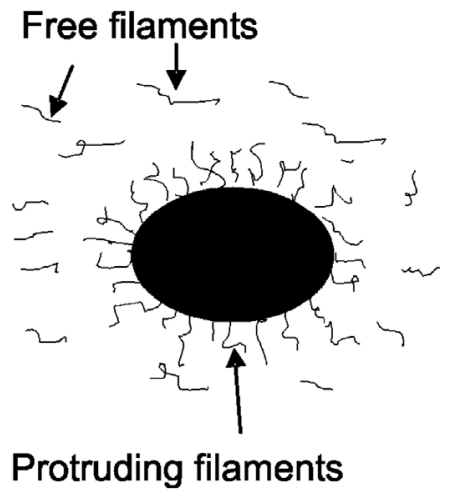

(b)

Figure 1. (a) Microscopic aggregates image. (b) Schematic representation of an aggregate and free filaments. Bar represents $100 \mu \mathrm{m}$. 


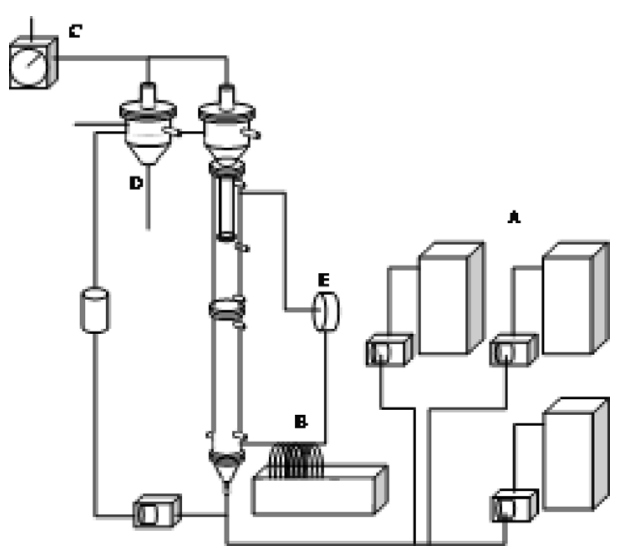

Figure 2. Experimental set-up. (A) Feed containers. (B) Water. (C) Biogas flow-meter. (D) External settler. (E) Water recirculation pump.

$6.5 \mathrm{~kg} \mathrm{COD} \mathrm{m}^{3} \mathrm{~d}$ and upflow liquid velocities, up to $6 \mathrm{~m} / \mathrm{h}$ were applied.

\section{Routine Analysis}

Routine reactor performance was monitored by determining influent and effluent total and soluble (centrifuged $10 \mathrm{~min}$ at $15000 \mathrm{rpm}$ ) chemical oxygen demand (COD), influent flow rate, $\mathrm{pH}$, and volatile suspended solids (VSS). Chemical oxygen demand and VSS were determined according to standard methods (APHA et al., 1989). The effluent VSS were determined by measuring periodically the total amount of VSS that accumulated in the external settler during a defined time period.

\section{Seed Sludge and Substrate}

The inoculum consisted of dispersed anaerobic biomass obtained from a local municipal sludge digester. Before inoculation it was screened by a $0.7-\mathrm{mm}$ sieve. The final volatile suspended solids content was $13.5 \mathrm{~g} \mathrm{SSV} \mathrm{L}$ and a volume of $5 \mathrm{~L}$ was added to the reactor. The influent $\mathrm{COD}$ was kept at $1500 \mathrm{mg}$ COD L by in-line diluting a concentrated synthetic feed, with tap water. In the first operation period (OP I), the feed was composed of $60 \%$ COD as glucose to promote the growth of acidogenic bacterial populations. The remaining 40\% COD were composed of acetate and propionate at a COD ratio of 70:30. In the second operation period (OP II) the glucose COD was reduced to $30 \%$, and the remaining $70 \%$ COD had a similar composition in acetate and propionate as in OPI. Sodium bicarbonate and calcium carbonate were used as alkalinity source. Micro- and macronutrients were added according to Zehnder et al. (1980).

\section{Specific Methanogenic Activity Assays}

The Specific Methanogenic Activity (SMA) assays were performed using a pressure transducer technique (Colleran et al., 1992). Specific methanogenic activity was measured against different individual substrates: acetate, propionate, butyrate, and $\mathrm{H}_{2} / \mathrm{CO}_{2}$. No calcium or trace-nutrients were added. Methane was measured by gas chromatography with helium as the carrier gas and a TCD detector.

\section{Scanning Electron Microscopy (SEM)}

Scanning Electron Microscopy (SEM) observations were carried out using a Leica-Cambridge S360 Scanning Electron Microscope equipped with an Oxford Instruments energy dispersion spectrograph (EDS). The samples were prepared according to the procedures described by Harper and Pohland (1997).

\section{Sludge Sampling and Processing for Image Analysis}

A sampler was designed to take biomass from the reactor without disturbing its morphology. The maintenance of granular integrity during sludge sampling and processing was a crucial step in this work. A wide-bore tube equipped with a valve was introduced by the top of the reactor and a sample of biomass present at 20 -cm height from the bottom, was collected, avoiding mechanical stress. Sludge samples for image analysis were taken once a week. For all the samples, the VSS content was determined.

Special care was put on the dilution process. Biomass samples must be diluted for image analysis using an optimized dilution factor. Excessive dilution increases the number of objects detected, due to a search-error practice. If the dilution is insufficient, the objects will be overlaid. Experiments were done to determine the optimal dilution value for the different measurements performed (filaments, micro and macro-aggregates). The optimal dilution value was determined as the lowest dilution that enabled the maximum percentage of objects recognition. The percentage of recognition is the ratio between the area of objects that are completely inside the image and the total area of objects in the image, including those that are at the boundaries and cannot be completely recognized.

\section{Image Acquisition and Analysis}

Filament image acquisition was accomplished through phase-contrast microscopy on a Diaphot 300 Nikon microscope (Nikon Corporation, Tokyo) with a $100 \times$ magnification. Images used to quantify aggregates larger than $0.2 \mathrm{~mm}$ in equivalent diameter were acquired through visualization on an Olympus SZ 40 stereo microscope (Olympus, Tokyo) with a $40 \times$ magnification. Images used to quantify aggregates smaller than $0.2 \mathrm{~mm}$ in equivalent diameter were acquired through visualization on a Zeiss Axioscop microscope (Zeiss, Oberkochen) with a $100 \times$ magnification. All the images were digitized and saved with the help of a CCD AVC D5CE Sony grey scale video camera (Sony, Tokyo) and a DT 3155 Data Translation frame grabber (Data Translation, Marlboro), with a $768 \times 576$ pixel size in 8 bits 
(256 grey levels) by the Image Pro Plus (Media Cybernetics, Silver Springs, MD) software package.

For the filaments and aggregates with an equivalent diameter smaller than $0.2 \mathrm{~mm}$, designated as micro-aggregates, a volume of $35 \mu \mathrm{L}$ from the diluted sample was dispensed on a slide and covered with a $24 \times 24 \mathrm{~mm}$ cover slip for visualization and image acquisition in phase contrast. This volume was selected to ensure that the drop was exactly covered by the cover slip, allowing computing the volume of $0.0499 \mu \mathrm{L}$ for each image. For the aggregates with an equivalent diameter larger than $0.2 \mathrm{~mm}$, designated as macro-aggregates, a volume of $2.8 \mathrm{~mL}$ was transferred to a Petri dish for visualization and image acquisition. Around 100 images per sample were acquired. Image acquisition of the micro-aggregates and filaments was obtained by three parallel horizontal passages along the slide, at $1 / 4,1 / 2$, and $3 / 4$ of its height, in a total of 24 images per slide. With respect to the larger aggregates, the Petri dish was thoroughly screened from left-to-right and top-to-bottom, making it possible to calculate the volume of $15.15 \mu \mathrm{L}$ for each image. For each magnification, the pixels were converted to metric dimensions using a micrometer.

The image analysis and processing was accomplished by means of three specially developed programs in Matlab (The Mathworks, Inc., Natick, MA), for filaments, micro and macro-aggregates.

\section{Filaments Program}

In this program the grey-scale image was first divided by the background image to remove background light differences. A bottom hat filter (Russ, 1995) was then applied to enhance the filaments and small aggregates that have low grey levels. The larger aggregates, which have high grey levels, were subsequently identified on the image resulting from the background elimination step, by consecutively applying a 10-order closing (to enhance the aggregates), a segmentation at a fixed threshold value, a filling of the resulting binary image (to remove the inner holes in the aggregates), and a erosion-reconstruction step to eliminate the debris. Filaments and small aggregates were then isolated by segmentation at a fixed threshold and by logic subtraction of the mask binary image containing the large aggregates. Then, the small aggregates were eliminated by deleting all the objects smaller than 32 pixels (in area) or with a gyration radius below 1.2. The final image contained only filaments that were characterized in terms of their length and number. The filaments were not only comprised of the dispersed bulk filaments, but also included those that were attached to an aggregate and still had one free extremity (Fig. 1). The filaments image was skeletonized and pruned (Russ, 1995) and then the filaments length was determined by:

$$
L=N \times 1.122 \times F_{\text {cal }}
$$

where $N$ is the number of pixels of the skeletonized filament and $F_{c a l}$ is the calibration factor $(\mu \mathrm{m} / \mathrm{pixel})$. The factor 1.1222 is used to homogenize the different angles of the filaments (Walsby and Avery, 1996). Finally, the specific total filament length was calculated as $L_{\text {spec }}=L / V_{\text {field }}$ where $V_{\text {field }}$ is the volume (in $\mu \mathrm{m}^{3}$ ) corresponding to the field of view (i.e., the image).

\section{Micro-Aggregates Program}

In this program the grey-scale image was first divided by the background image to remove background light differences. Then, a histogram equalization was performed to enhance the micro-aggregates, followed by an image smoothing Wiener filtering. The image was then segmented in black (background) and white (objects), by the simultaneous use of a boundary- based segmentation and a user chosen or automatically determined threshold segmentation. The objects smaller than $3 \times 3$ pixels (small debris) were then removed and small gaps $(6 \times 6$ pixels or less $)$ were filled on the remaining objects. Subsequently, to remove filaments, all the objects smaller than 2000 pixels in area and with a gyration radius above 1.2 were deleted. Finally, all the objects cut off by the image boundaries were removed, and the morphological characterization of the micro-aggregates was performed.

\section{Macro-Aggregates Program}

In this program the grey-scale image was first divided by the background image to remove background light differences, followed by an image-smoothing Wiener filtering. The image was then segmented in black (background) and white (aggregates), by threshold segmentation, with a user chosen or automatically determined threshold. Subsequently, small gaps $(6 \times 6$ pixels or less) were filled on the objects, and small debris $(3 \times 3$ pixels or less) was removed. Finally, all the objects cut off by the image boundaries were removed, and the morphological characterization of the macroaggregates was performed. Figure 3 represents original and binary images obtained from the three programs.

The parameters determined by these two last programs are the aggregate area from which the equivalent diameter is calculated:

$$
D_{e q}=2 \cdot F_{\text {cal }} \sqrt{\text { Area } / \pi}
$$

and the total area $\left(A_{T}\right)$ occupied by aggregates in each image from which the specific area occupied by aggregates is calculated:

$$
A_{\text {spec }}=A_{T} / V_{\text {field }}
$$

Finally, a morphological parameter based on the ratio of specific total filament length to total projected area of aggregates $(L f A)$ is determined:

$$
L f A=L_{\text {spec }} /\left(A_{\text {spec }(<0.2 m m)}+A_{\text {spec }(\geq 0.2 m m)}\right.
$$




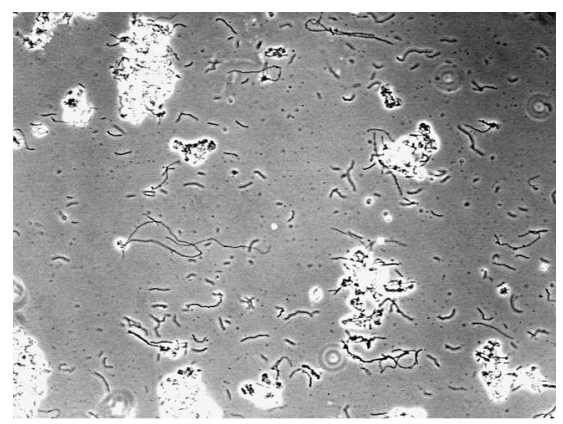

(a)

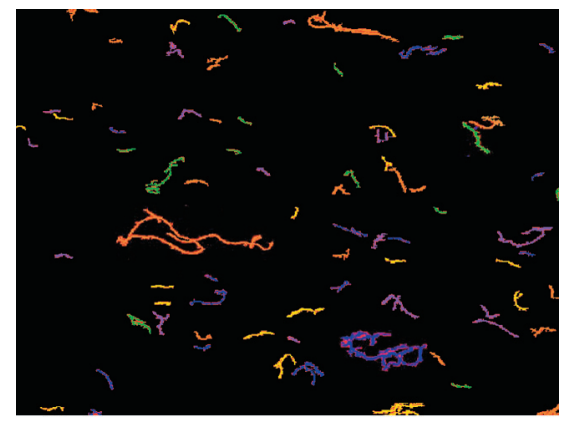

(d)

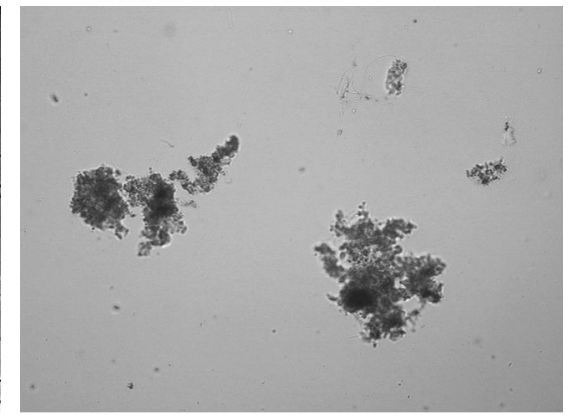

(b)

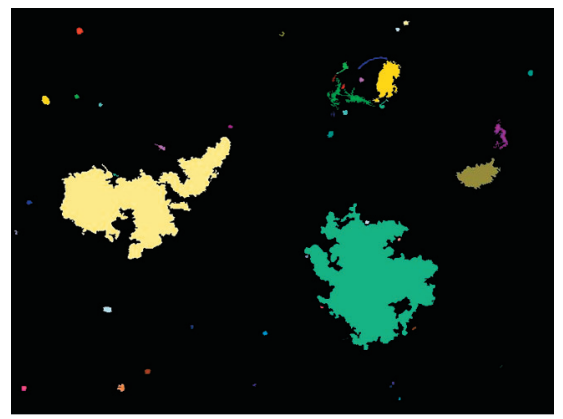

(e)

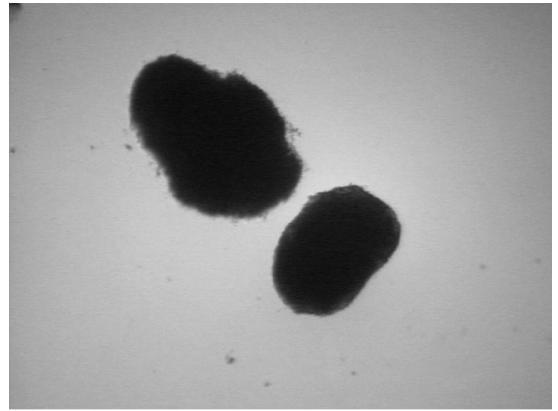

(c)

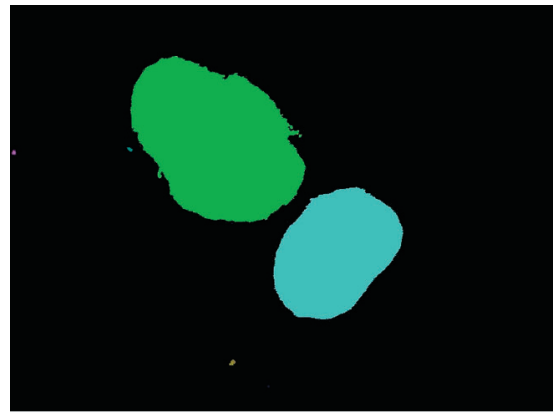

(f)

Figure 3. Original images of filaments, micro-aggregates, and macro-aggregates (a, b, and c) and the respective final binary images (d, e, f).

where $A_{\text {spec }(<0.2 \mathrm{~mm})}$ and $\left.A_{\text {spec }} \geq 0.2 \mathrm{~mm}\right)$ are the specific aggregate area ratio for aggregates of equivalent diameter $<0.2 \mathrm{~mm}$ and $\geq 0.2 \mathrm{~mm}$, respectively.

\section{RESULTS AND DISCUSSION}

\section{EGSB Operation}

Chemical oxygen demand removal in the EGSB reactor was consistently above $90 \%$ throughout all the experiment (Fig. 4a). The upflow velocity, which never exceeded $3 \mathrm{~m} / \mathrm{h}$ during the first operation, was increased to $5.7 \mathrm{~m} / \mathrm{h}$ for the second run (Fig. 4b). The $\mathrm{pH}$ in the reactor remained in the range $6.42-7.41$, with an average value of $6.91 \pm 0.21$.

\section{Image Analysis}

The monitoring of the growth of the aggregates through the corresponding average equivalent diameter was unsuccessful, since this parameter largely oscillated throughout the operating period. Different size classes were then studied: aggregates ranging from an equivalent diameter of $0.0038 \mathrm{~mm}$ (minimal size of the aggregates recognized by the image analysis program) up to $0.01 \mathrm{~mm}$, designated as residual aggregates; aggregates ranging from an equivalent diameter of $0.01 \mathrm{~mm}$ up to $0.1 \mathrm{~mm}$, aggregates ranging from an equivalent diameter of $0.1 \mathrm{~mm}$ up to $1 \mathrm{~mm}$, and finally aggregates with equivalent diameters larger than $1 \mathrm{~mm}$. Figure 5 presents the average equivalent diameters within each size class during the two operation periods.
A granule is considered as a structure built up after nucleation and aggregation that ideally maintains a stable morphology while evolving with time due to the dynamics between growth, erosion, and fragmentation. The evolution of the ratio between the total filament length and the total aggregate area $(L f A)$ is presented in Figure 6a for OP I. The changes in the $L f A$ value are related to the balance between

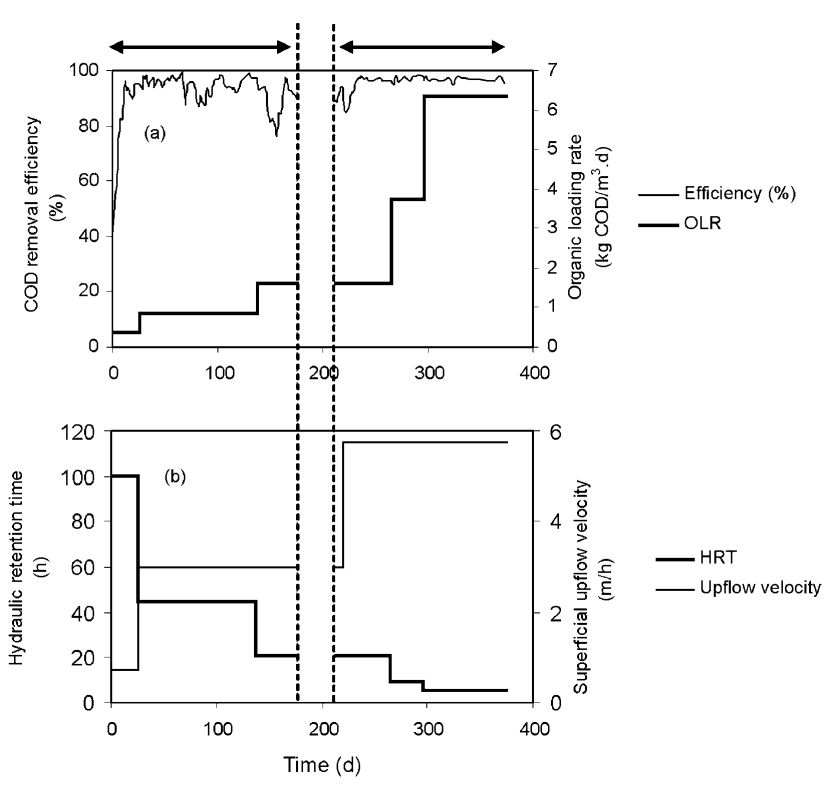

Figure 4. (a) Time course of COD removal efficiency and organic loading rate (OLR). (b) Superficial velocity (SV) and hydraulic retention time (HRT). 


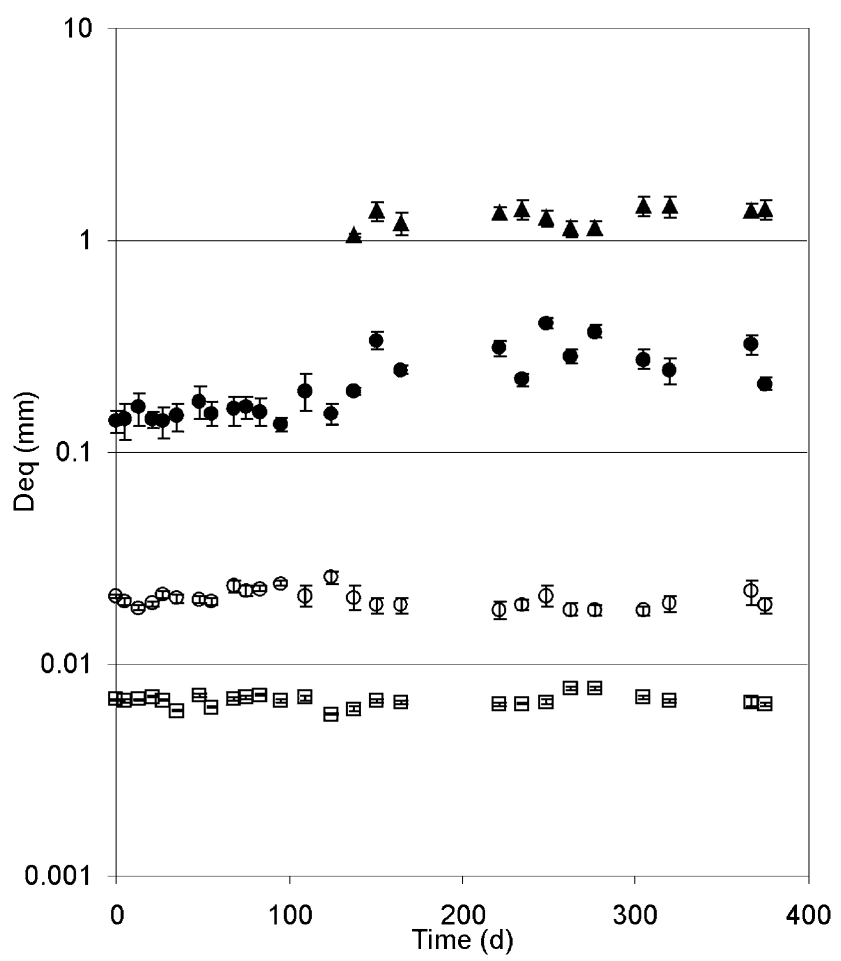

Figure 5. Aggregates equivalent diameter throughout the granulation process for the different size classes. $(\boldsymbol{\Lambda})$ equivalent diameter $>1 \mathrm{~mm},(\bullet)$ equivalent diameter between 0.1 and $1 \mathrm{~mm},(\bigcirc)$ equivalent diameter between 0.01 and $0.1 \mathrm{~mm},(\square)$ equivalent diameter $<0.01 \mathrm{~mm}$.

the filaments abundance (as presented in Fig. 1b) and the total area of aggregates. In the first stages of granulation, the enrichment on filamentous forms when the projected areas are still small, led to the increase of $L f A$ values. After this initial increase, and regardless of the intrinsic evolution of both parameters involved in the $L f A$ definition, there are two remarkable events traduced by significant changes in $L f A$. The first one, when LfA starts to decrease around day 50, suggests a change in the dynamic evolution of filaments and aggregates, possibly due to the washout of the free filaments and/or growth in diameter and consistency of the aggregates. The second event is when $L f A$ stops decreasing and becomes stable, revealing that the growing granule has achieved a state where the above-mentioned growth dynamics is established. This last instance can be defined as the aggregation time $\left(\theta_{\mathrm{a}}=110\right.$ days).

The dynamics of aggregation may also be described by the percentage of total projected area of aggregates within each size range (Fig. 6b). Although it was observed that very small aggregates in the size range $0.01-0.1 \mathrm{~mm}$ represented more than $90 \%$ in number of particles, after reaching the aggregation time $\theta_{\mathrm{a}}$, the aggregates in the size range $0.1-$ $1 \mathrm{~mm}$ represented about $90 \%$ of the total projected area of aggregates. At that time, no aggregates larger than $1 \mathrm{~mm}$ (equivalent diameter) were detected, but after the day 137 a number of particles larger than $1 \mathrm{~mm}$ started to appear in the reactor. The increase in the relative abundance of those particles was simultaneous with the decrease in the per-
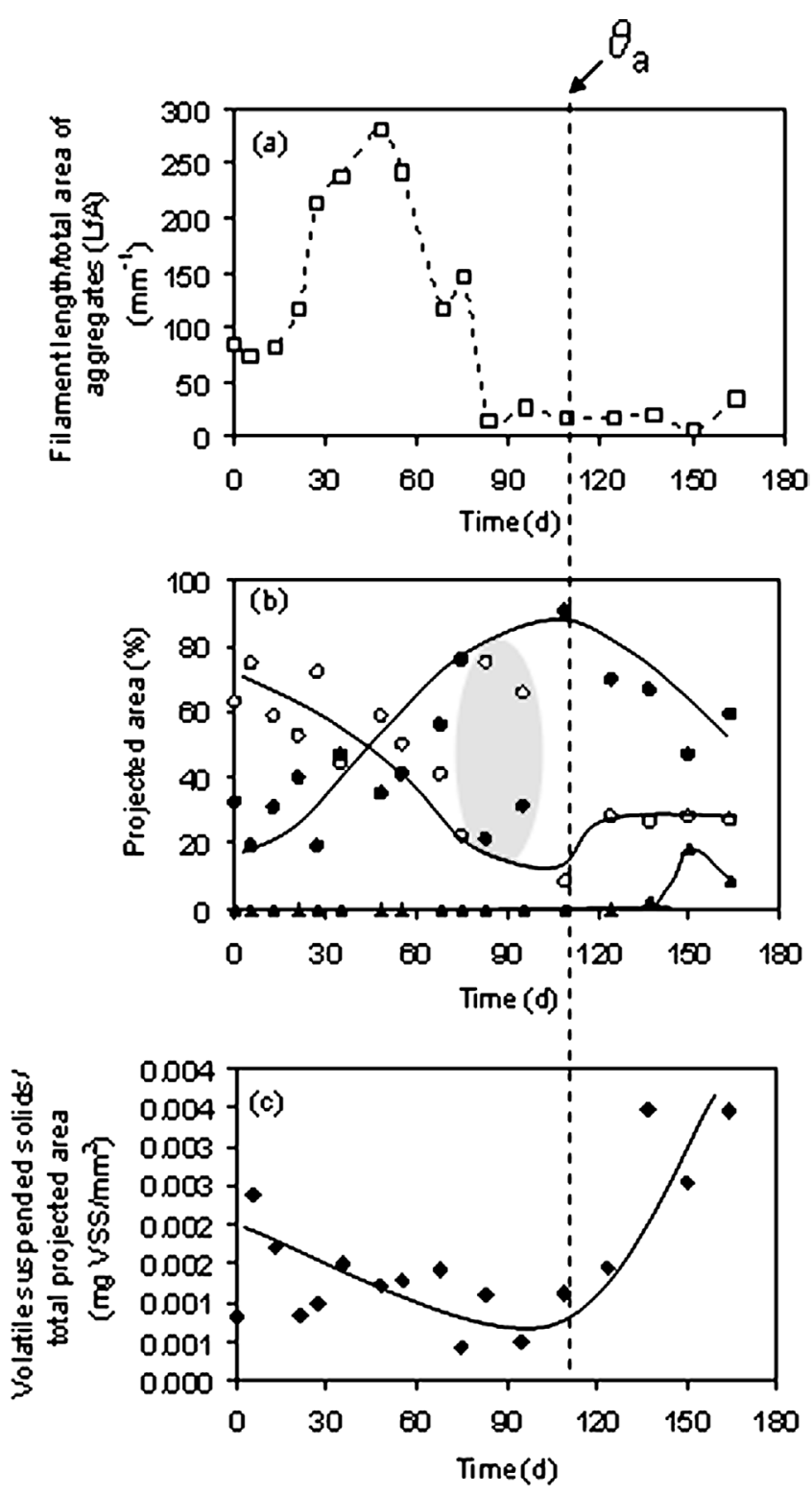

Figure 6. (a) Time course of total filament length/total aggregate area $(L f A),(\mathbf{b})$ percentage of projected area for different size classes, and (c) volatile suspended solids per projected area unit, during the OP Igranulation period. ( $\Delta$ ) equivalent diameter $>1 \mathrm{~mm},(\bullet)$ equivalent diameter between 0.1 and $1 \mathrm{~mm},(\mathrm{O})$ equivalent diameter between 0.01 and $0.1 \mathrm{~mm}$.

centage corresponding to the particles within the range $0.1-1 \mathrm{~mm}$. A slight increase in the particles $0.01-0.1 \mathrm{~mm}$ was also observed, which could account for the growth of newly formed aggregation nuclei. Considering that the projected area may be related to the biomass quantity, this clearly evidences that after $\theta_{\mathrm{a}}$, the sludge became structured into growing aggregates. However, a linear relationship between the projected area and the VSS content is not possible, because the VSS are related to volume and not to area and because aggregates density may change with time. This can be observed in Figure 6c, where the VSS per projected area unit is presented, showing a remarkable increase after the aggregation time $\theta_{\mathrm{a}}$. 

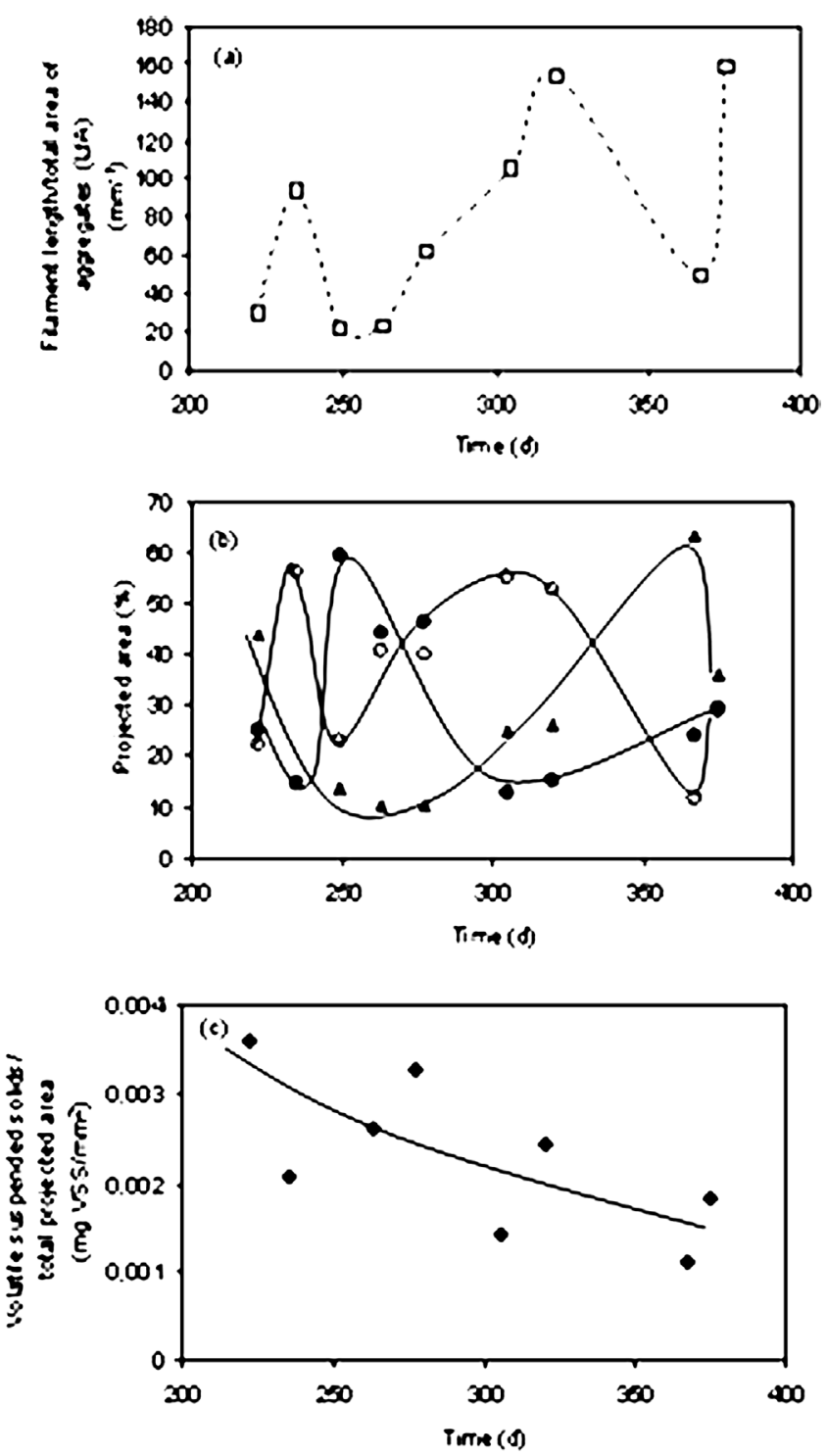

Figure 7. (a) Time course of total filament length/total aggregate area (LfA), (b) percentage of projected area for different size classes, and (c) volatile suspended solids per projected area unit, during the OP IIgranules maturation period. ( $\mathbf{\Delta})$ equivalent diameter $>1 \mathrm{~mm},(\bullet)$ equivalent diameter between 0.1 and $1 \mathrm{~mm},(\mathrm{O})$ equivalent diameter between 0.01 and $0.1 \mathrm{~mm}$.

Figure 7 describes the effect of changes in organic loading rate and upflow velocity on the established granular sludge (OP II). The two peaks obtained for the LfA parameter on days 235 and 320 , may be related to the increase on upflow velocity on day 220 and to the increases on OLR on days 265 and 296 (Figs. 4b and 7a). Higher upflow velocities impose higher shear stress on the granules surface. Filaments that are loosely adhered to the aggregates and/ or that are growing out of the aggregates can detach, increasing the total detectable filament length. On the other hand, a phenomenon of erosion may reduce the aggregates size, increasing the amount of small fragments. The increase on the organic loading rate induces an increase on biogas production that may not be easily released out of the granule and therefore may promote some type of fragmentation. The occurrence of erosion and/or fragmentation seems to be reasonable when comparing Figure $7 \mathrm{a}$ and $7 \mathrm{~b}$, where a coincidence is observed, between the peaks on $L f a$ and on the \% of projected area of the smaller aggregates $(0.01-0.1 \mathrm{~mm}$ equivalent diameter). This clearly shows the level of granules deterioration likely induced by the sudden increases on OLR on days 265 and 296. The fluctuations in the percentage of projected area between the three size classes are evident all along this period, and only at the end of the operation did the aggregates larger than $1 \mathrm{~mm}$ start to dominate the total projected area. The VSS per projected area decreased continuously along the OP II, which may result from a loss of sludge density (Fig. 7c).

The increase in the LfA was related to an increase in washout, detected by the increase on effluent VSS values. On day 235, the average amount of sludge (on a 3-d basis) that accumulated on the external settler was $0.44 \mathrm{~g}$ VSS d, but on days 305 and 320, this amount increased to 4.25 and $4.43 \mathrm{~g}$ VSS d, respectively. Taking into account the flow rate through the settler, the average effluent VSS concentration could be estimated, as presented in Figure 8 along with the LfA values. It is evident that, in general, the increase in LfA accompanied the increase in washout. This can be important from a practical viewpoint: although more experiments should be performed, there are hints that $L f A$ could be used for an early alert of washout events.

The results obtained during OP II show the structural and morphological sludge dynamics, and evidence of the occurrence of filaments release and fragmentation, likely resulting from granules erosion induced by the step increases in the upflow velocity and in the organic loading rate. These phenomena have already been described in a qualitative way or, alternatively, quantification was essentially based on effluent VSS measurements. The added value of the present work is to provide an accurate methodology to quantitatively describe them, giving new insights about granular sludge stability inside EGSB reactors.

\section{Microscopic Observations}

Figure 9 represents acquired images to quantify filaments and macro-aggregates from sludge samples taken on days

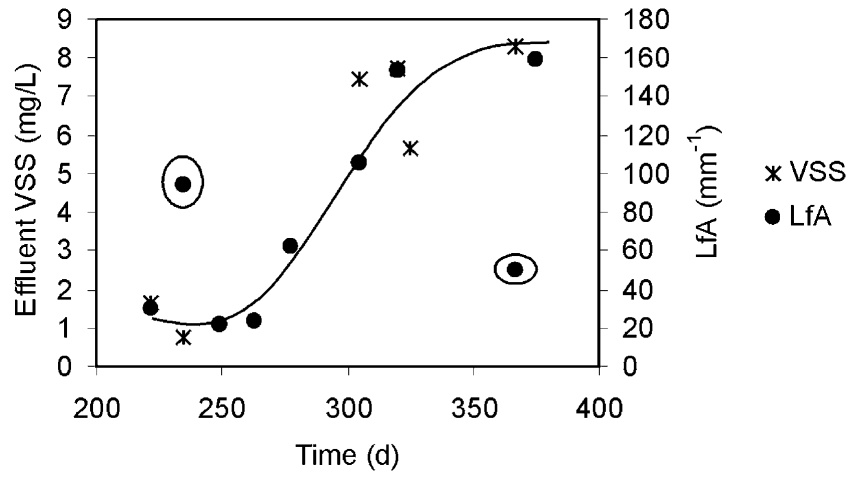

Figure 8. Time course of effluent VSS and LfA along the OP II. 

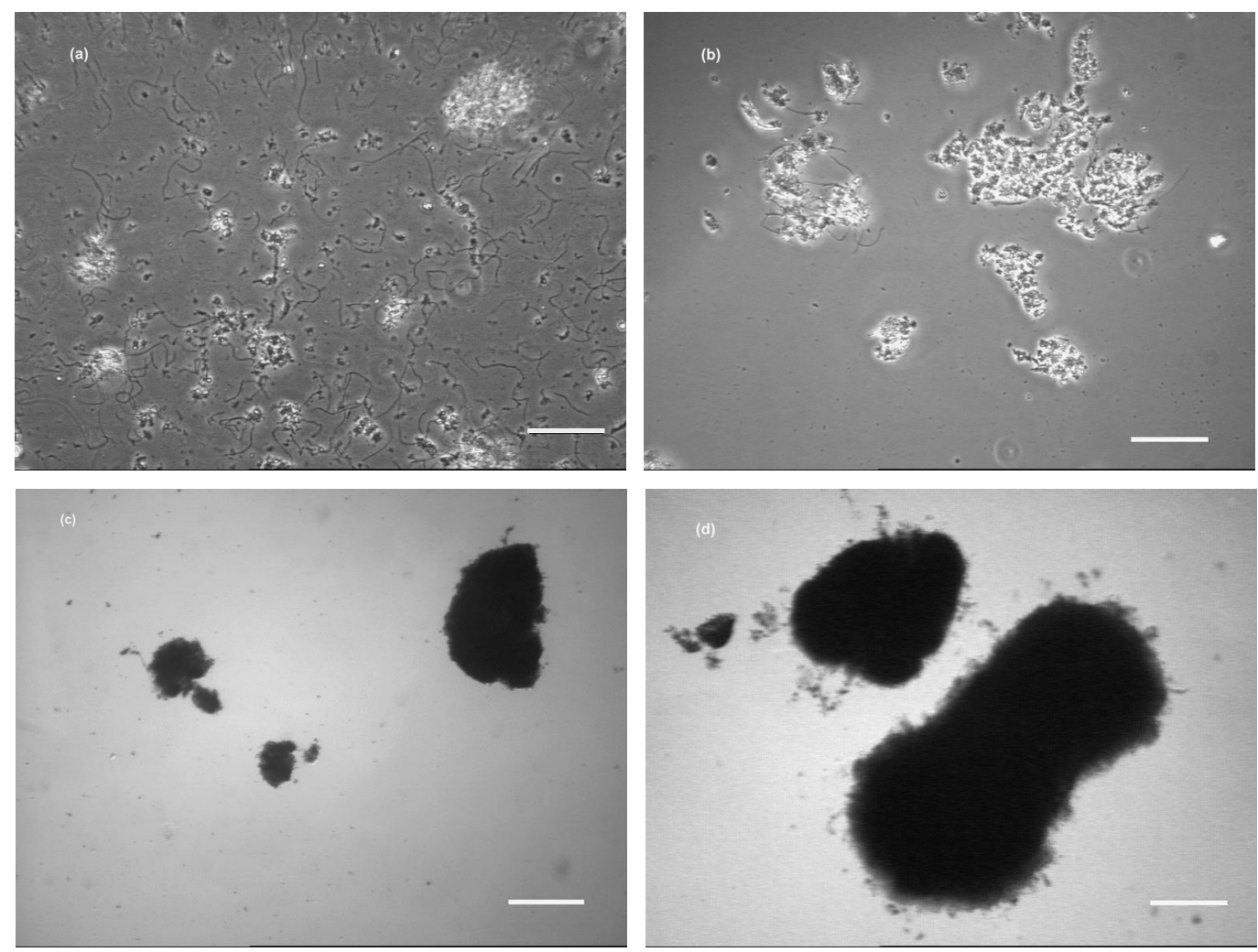

Figure 9. Examples of acquired images for measuring filaments, taken on days 48 (a) and 83 (b), (OP I) and examples of images acquired for measuring macro-aggregates, taken on days 222 (c) and 375 (d) (OP II). The bar represents $100 \mu \mathrm{m}$ for images (a) and (b) and $500 \mu \mathrm{m}$ for images (c) and (d).

48 and 83 (images for filaments quantification in OP I) and on days 222 and 375 (images for macro-aggregates quantification in OP II). Comparing Figures $9 \mathrm{a}$ and $9 \mathrm{~b}$ it is understandable the different values of $L f A$ obtained-282.9 and 16.1, respectively. The increase in size of the aggregates higher than $1 \mathrm{~mm}$ was also evident, and on day 375 a rough surface can be observed, due to the excessive growth of filamentous bacteria (Fig. 9d).
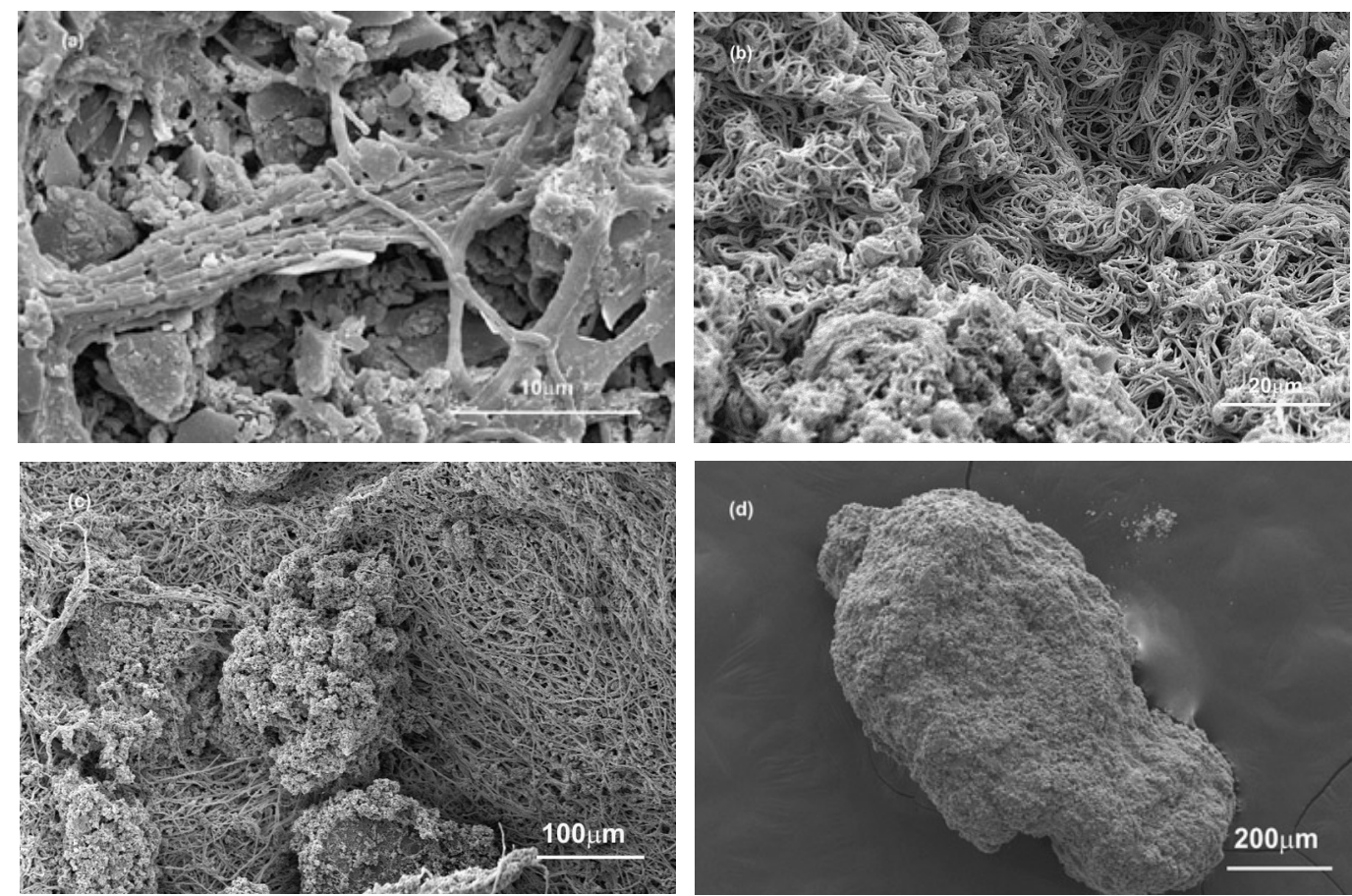

Figure 10. SEM photographs from different stages of the operation period: inoculum (a), day 62 (b), day 90 (c), day 222 (d). 
Scanning electron microscopy (SEM) was used to visualize the surface of aggregates along the experiment (Fig. 10).

The inoculum was a completely dispersed biomass mixed with other fine solids present in the sludge. Figure 10a shows its heterogeneity, consisting mainly of residual mineral fragments, and a diversity of microorganisms. Figure 10b represents a sample from day 62 and shows a remarkable change in biomass composition where the filamentous bacteria and the intricate matrix that is being formed to become the backbone of the granules structure can be clearly distinguished. The compaction of this structure can be seen in Figure 10c were the previously formed matrix shows less dead spaces as well as the incorporation of calcium and calcium bicarbonate crystals (sample from day 90). The energy dispersion spectroscopy analysis showed an increase in the calcium content of the aggregates. This mineralization phenomenon contributed to the settling characteristics of the granules and to the development of a $\mathrm{pH}$ protecting microenvironment, which are important features of a mature granule as the one observed in Figure $10 \mathrm{~d}$, collected at the day 222 .

\section{Methanogenic Activity}

Figure 11 presents the values of the specific methanogenic activity measured along the experiment.

Concerning the methanogenic activity against propionate and butyrate as substrates it should be said that, since these substrates are indirect methanogenic substrates, a valid measurement of the maximum specific methanogenic activity against these acids, can only be obtained when the acetoclastic and hydrogenophilic activities are not ratelimiting (Dolfing and Bloemen, 1985). In the present situation this condition prevailed in all cases.

The increase in the upflow liquid velocity at day $220 \mathrm{did}$ not affect significantly the SMA, probably because external mass transfer was not limiting the access of substrate to the

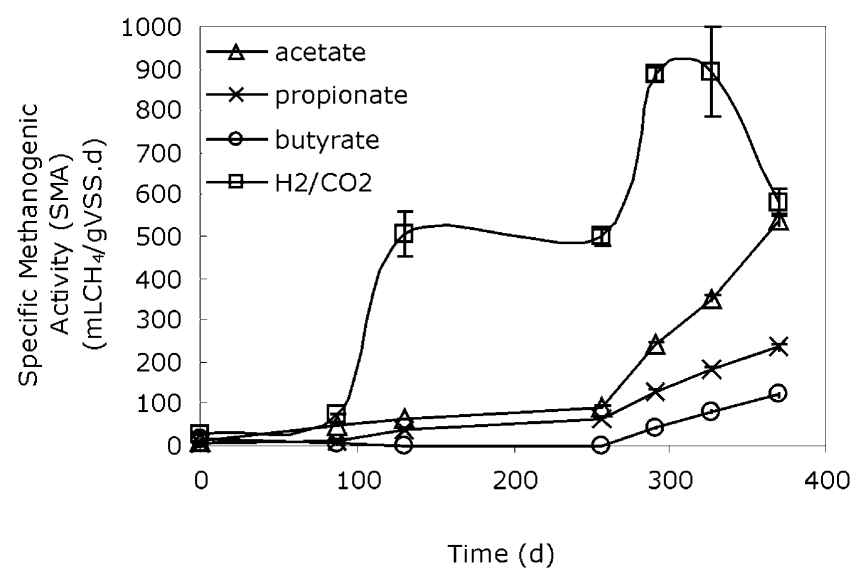

Figure 11. Time course of the specific methanogenic activity with acetate, propionate, butyrate, and $\mathrm{H}_{2} / \mathrm{CO}_{2}$ as substrates. sludge in the former conditions. On day 265 the OLR value was increased to $4 \mathrm{kgCOD} \mathrm{m^{3 }} \mathrm{d}$ and this induced a considerable increase in all the SMA measured at day 291, which can be attributed to the higher load of substrate supply. The SMA on acetate, propionate, and butyrate increased steadily between days 256 and 376, while the hydrogenophilic activity suddenly increased after day 110 , which was the critical moment designated by aggregation time $\left(\theta_{\mathrm{a}}\right)$. This increase is in accordance with the need for interspecies hydrogen transfer and underlines the important role of hydrogenophilic bacteria in the granulation process. According to Gujer and Zehnder (1983) there is a maximum distance between syntrophic bacteria that allows hydrogen to be transferred, while Schink and Thauer (1988) emphasized the importance of syntrophic relationships, considering that they are the driving force for granulation.

\section{CONCLUSIONS}

The development of image analysis techniques allowed the quantitative monitoring of the dynamic changes of aggregates along the anaerobic granulation process and the identification of critical moments such as the aggregation time. A parameter defined as the ratio filament length/total aggregate area $(L f A)$ has proven to be sensitive to changes in the aggregation status of the anaerobic sludge. $L f A$ allowed the recognition of the aggregation time and accompanied the trend of biomass washout. Other measurements such as the percentage of projected area of aggregates within three size classes $(0.01-0.1 \mathrm{~mm}, 0.1-1 \mathrm{~mm}$, and $>1 \mathrm{~mm}$, equivalent diameter) and a parameter defined as the volatile solids content per projected area unit, reflected the aggregation/granules maturation phenomena. All along the operation of the EGSB reactor, the specific methanogenic activity (SMA) was raised over initial values. A significant increase in the hydrogenophilic activity was detected immediately after the aggregation time, which emphasizes the importance of interspecies hydrogen transfer for the granulation phenomena. The SEM observations demonstrated that the formed structures present a set of attributes such as homogeneity and microbial complexity consistent with their description as granules.

The results show, in a quantitative way, the structural and morphological sludge dynamics during a process of granulation and demonstrate the usefulness of the proposed parameters to describe the occurrence of filaments' release and fragmentation resulting from granules erosion induced by changes in the upflow velocity and on the organic loading rate. This methodology can give new insights about granular sludge stability inside EGSB reactors. A second example is given in Part II (Amaral et al., this issue) with the monitoring of a granule deterioration process triggered by the contact with oleic acid.

We grateful acknowledge the financial support to P. Araya-Kroff, A.L. Amaral, and L. Neves through the grants SFRH/BD/3187/ 2000, PRAXIS XXI/BD/20325/99 and POCTI/1999/CTA736524, 
respectively, from the Fundação para a Ciência e a Tecnologia (Portugal). The ICCTI (Portugal) and Ambassade de France in Portugal provided financial support for cooperation between Braga and Nancy teams through project no. 203 B4.

\section{References}

Alves MM, Cavaleiro AJ, Ferreira EC, Amaral AL, Mota M, da Motta M, Vivier H, Pons M-N. 2000a. Characterization by image analysis of anaerobic sludge under shock conditions. Wat Sci Technol 41: $207-214$.

Alves MM, Ferreira EC, Amaral AL, Pereira MA, Novais JM, Mota M. 2000b. Staged and non-staged anaerobic filters: Performance in relation with the physical and biological characteristics of microbial aggregates. J Chem Technol Biotechnol 75:601-609.

Amaral AL, Pereira MA, da Motta M, Pons M-N, Mota M, Ferreira EC, Alves MM. 2004. Development of image analysis techniques as a tool to detect and quantify morphological changes in anaerobic sludge: II. Application to a granule deterioration process triggered by the contact with oleic acid. Biotechnol Bioeng 87:194-199.

APHA, AWWA, WPCF. 1989. Standard methods for the examination of water and wastewater, 17th ed. Washington DC: American Public Health Association.

Colleran E, Concannon F, Goldem T, Geoghegan F, Crumlish B, Killilea E, Henry M, Coates J. 1992. Use of methanogenic activity tests to characterize anaerobic sludges, screen for anaerobic biodegradability and determine toxicity thresholds against individual anaerobic trophic groups and species. Wat Sci Technol 25:31-40.

Dolfing J, Bloemen WGBM. 1985. Activity measurements as a tool to characterize the microbial composition of methanogenic environments. J Microbiol Meth 4:1-12.

ElMamouni R, Leduc R, Costerton JW, Guiot SR. 1995. Influence of the microbial content of different precursory nuclei on the anaerobic granulation dynamics. Wat Sci Technol 32:173-177.

Frankin RJ. 2001. Full-scale experiences with anaerobic treatment of industrial wastewater. Wat Sci Technol 44:1-6.

Gujer W, Zehnder AJB. 1983. Conversion processes in anaerobic digestion. Wat Sci Technol 15:127-167.

Harper RS, Pohland FG. 1997. Microbial consortia selection in anaerobic filters operated in different reactor configurations. Wat Sci Technol 36:33-39.

Hulshoff Pol LW. 1989. The phenomenon of granulation of anaerobic sludge. Ph.D. Thesis, Wageningen Agricultural University, The Netherlands.

Jenkins D, Richard MG, Daigger GT. 1993. Manual on the causes and control of activated sludge bulking and foaming. Boca Raton, FL: Lewis Publishers.

Lettinga G, Hulshoff Pol LW. 2002. The sludge granulation phenomenon. Its augmentation, manipulation and challenges (part 2). Proceedings of Granulation and Auto-immobilisation Processes in Wastewater Treatment. Wageningen, The Netherlands: Lettinga Association Foundation p 97-109.

MacLeod FA, Guiot SR, Costerton JW. 1990. Layered structure of bacterial aggregates produced in an upflow anaerobic sludge bed and filter reactor. Appl Env Microbiol 56:1598-1607.

Morvai L, Mihaltz P, Czako L. 1992. The kinetic basis of a new start-up method to ensure the rapid granulation of anaerobic sludge. Wat Sci Technol 25:113-122.

Russ CR. 1995. The image processing handbook. Boca Raton, FL: CRC Press.

Schink B, Thauer RK. 1988. Energetics of syntrophic methane formation and the influence of aggregation. In: Lettinga G, Zehnder AJB, Grotenhuis JTC, Hulshoff Pol LW, editors. Proceedings of Granular Anaerobic Sludge: Microbiology and Technology (GASMAT) Workshop. The Netherlands: Puduc Wageningen. p 5-17.

Singh RP, Kumar S, Ojha CSP. 1998. A critique on operational strategies for start-up of UASB reactors: Effects of sludge loading rate and seed/ biomass concentration. Biochem Eng J 1:107-119.

van Lier JB, Tilche A, Ahring BK, Macarie H, Moletta R, Dohanyos M, Pol LWH, Lens P, Verstraete W. 2001. New perspectives in anaerobic digestion. Wat Sci Technol 43:1-18.

Walsby AE, Avery A. 1996. Measurement of filamentous cyanobacteria by image analysis. J Microbiol Meth 26:11-20.

Wiegant W, de Man A. 1986. Granulation of biomass in thermophilic upflow anaerobic sludge blanket reactors treating acidified wastewaters. Biotechnol Bioeng 28:718-727.

Zehnder AJB, Huser BA, Brock TD, Wuhrmann K. 1980. Characterization of an acetate-decarboxylating, non-hydrogen-oxidizing methane bacterium. Arch Microbiol 124:1-11. 